\title{
The QTL Mapping of the Important Breeding Traits in Winter Triticale $(\times$ Triticosecale Wittm.)
}

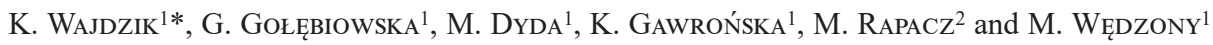 \\ ${ }^{1}$ Institute of Biology, Department of Cell Biology and Genetics, Pedagogical University of Cracow, \\ Podchorążych 2, Kraków 30-084, Poland \\ ${ }^{2}$ Department of Plant Physiology, University of Agriculture in Krakow, Kraków, Poland
}

(Received 19 June 2018; Accepted 13 May 2019;

Communicated by A. Goyal)

\begin{abstract}
The increasing economic importance of triticale ( $\times$ Triticosecale Wittm.) makes this synthetic hybrid cereal an interesting object of genetic studies. Genomic regions (QTL) of morphological winter triticale traits were determined using the mapping population of 89 doubled haploids lines (DHs) developed from $\mathrm{F}_{1}$ hybrid of cv. 'Hewo' and cv. 'Magnat' accompanied with the genetic map consisting of 20 linkage groups assigned to the A (7), B (7), and R (6) genomes (total of 3539 DArT, SNP-DArT and SSR markers, length of $4997.4 \mathrm{cM})$. Five independent experiments were performed in the field and greenhouse controlled conditions. A total of 12 major QTLs located on 2B, 5A, 5R, and 6B chromosomes connected to the stem length, the plant height, the spike length, the number of the productive spikelets per spike, the number of grains per spike, and the thousand kernel weight were identified by a composite interval mapping (CIM).
\end{abstract}

Keywords: QTL, triticale, morphological traits, yield components, genetic map

\section{Introduction}

Triticale ( $\times$ Triticosecale Wittm.) is a synthetic hybrid resulted from the crossing of hexaploid wheat (Triticum aestivum L., AABBDD) with cultivated rye (Secale cereale L., RR) and the subsequent duplication of chromosome numbers in the $\mathrm{F}_{1}$ hybrid. Derived in this way, octoploid (AABBDDRR) is genetically not stable and D genome chromosomes are predominantly being lost during the further breeding processes. Thus, a relatively stable and the most commonly grown triticale is hexaploid with a genomic constitution of $2 n=6 \mathrm{x}=42$ chromosomes (AABBRR) (Ammar 2004). This complex origin results in frequent homological and homological translocations of chromosomal fragments between component genomes (Lukaszewski 2003; Tyrka et al. 2011, 2015; Niedziela et al. 2012; Machczyńska et al. 2014).

Triticale raises the interest of breeders and farmers, because it can tolerate adverse environmental conditions while producing a reasonable yield of good quality as reviewed by Blum (2014). Moreover, its grain is high in essential amino acids (Strang et al. 2016) and it has capacity to tolerate acidic soils and high aluminium concentration (Niedziela

*Corresponding author; E-mail: kwajdzikup@gmail.com; Phone: +48 126627832 
et al. 2012). Triticale is not only a promising cereal but a valuable genetic bridge for transferring desirable genes among rye and wheat (Tyrka and Chełkowski 2004; Badea et al. 2011). Long lasting efforts to map the wheat genome made possible the localisation of QTLs of complex traits in its genome (Börner et al. 2002). Earlier maps of the rye genome (Korzun et al. 1996) have been lately completed with high density DArT maps (Gawroński et al. 2016). These circumstances helped to assemble triticale maps compiling various markers (Badea et al. 2011; Alheit et al. 2014; Tyrka et al. 2011, 2015, 2018). Data on triticale QTL analyses are scarce so far (Niedziela et al. 2012; Alheit et al. 2014). There is a need of an immense effort in this field to fill up the gap in our understanding of the cooperation of A, B, and R genome components in shaping the triticale phenotype.

The aim of the study was to locate regions of the genome (QTL) associated with the control of important, polygenic agronomic traits in winter triticale $(\times$ Triticosecale Wittm.). The newly established DH mapping population (Tyrka et al. 2015) enabled multiple repeating experiments in various environmental conditions. As a result, we have located significant QTLs for the stem length and plant height, the spike length, and the numbers of spikelets per spike and grains per spike as well as the thousand kernel weight.

\section{Materials and Methods}

\section{Plant material and experimental design}

The mapping population of 89 triticale $\mathrm{DH}$ lines derived from $\mathrm{F}_{1}$ hybrid of cv. 'Hewo' (Strzelce Plant Breeding - IHAR Group Ltd., Poland) as the female parent and cv. 'Magnat' (DANKO Plant Breeders Ltd., Poland) as the pollen donor, accompanied by a genetic triticale map consisting of 20 linkage groups assigned to the A (7), B (7), and R (6) genomes. The total length of the map is $4997.4 \mathrm{cM}$, and it contains 842 DArT, 2647 SNPDArT and 50 SSR markers (Tyrka et al. 2015). The material was available for the experiments at the Institute of Plant Physiology Polish Academy of Science (IPP PAS) in Kraków, Poland. All experiments were performed in completely randomized block design (CRBD).

Experiment 1 (2010/2011) was performed in controlled conditions. Grain of DH and the parental lines of the population were planted and vernalized in multi-pots according to Gołębiowska and Wędzony (2009). The vernalized plants were transplanted to Mitscherlich pots ( 5 seedlings per pot $20 \varnothing \mathrm{cm}$ ) with a mixture of soil/turf substrate/sand $(2 / 3 / 1, \mathrm{v} / \mathrm{v} / \mathrm{v})$ and grown in greenhouse conditions: $20-25^{\circ} \mathrm{C}, 8 \mathrm{~h} / 16 \mathrm{~h}$ (day/night) photoperiod. Plants were watered daily and supplemented with Hoagland and Arnon's (1938) sterile medium twice-a-week until harvesting.

Experiments: 2, 3 and 4 (2010/11, 2011/12, 2012/13, accordingly) were carried out in the experimental plots of IPP PAS. Kernels were sown by 7 (Experiment 2) and 10 (Experiments 3 and 4) per row with $20 \mathrm{~cm}$ plants spacing. Rows were ordered in a random design and spaced by $40 \mathrm{~cm}$. Both parental lines were sown every ten rows. Experimental plots were protected with netting against birds. 
Experiment 5 (2013/2014) was conducted in the experimental plots in Choryń (Danko Breeding Ltd.). Seeds were provided by IPP PAS. Three rows per DH, 20 kernels per row, $1 \mathrm{~m}$ in length with $25 \mathrm{~cm}$ distance, were sown in a randomized design. Both parental lines were sown in one row each every twenty rows.

\section{Phenotyping}

All measurements were made at the time when plants achieved harvest maturity. In Experiments 1-4 (under controlled conditions), the three highest shoots were evaluated for the stem length (STL) of each plant separately. The stem length (STL) was measured from the crown to the base of the spike (Experiments 1-4). The spike length (SL) was measured from the base of the spike to the base of the highest spikelet. All fertile spikelets contributed to the number of spikelets per spike (NSS), and all grains in a spike contributed to the calculation of the number of grains per spike (GS) and the thousand kernel weight (TKW).

In Experiment 5 (in field conditions), plant height (PH) was measured with a scaled rod in two random places in each row of every DH and the parental lines. The measurements were taken from the soil level to the top of the spike. NSS was made for three shoots selected randomly in each row. Remaining parameters were not evaluated in Experiment 5.

Data obtained from measurements all traits per each genotype were used to calculate the mean value, which the mean value (shown in Table 1) was taken for QTL analysis.

\section{Statistical analysis and QTL mapping}

For mean values of all measured traits, Shapiro-Wilk test was performed to verify normal distribution of data as well as analysis of skewness and kurtosis. ANOVA for a single experiment and Pearson's correlation were calculated for phenotypic data by using Statistica software v.12.0 (StatSoft, Inc. USA). A post-hoc comparison was conducted by Duncan's multiple range tests $(p<0.05)$. QTLs were identified using Windows QTLCartographer 2.5 software (Wang et al. 2007) by a Single Marker Analysis (SMA) and Composite Interval Mapping (CIM). The SMA analysis was used to detect the chromosome location in which QTLs were included. The CIM analysis was performed to confirm the chromosome region associated with the morphological traits evaluated. Significance at the $p=0.05$ level was determined from 1000 permutations and LOD threshold $\geq 3.0$ to detect QTL regions described as a result of the present work. The QTLs for a particular trait with identical, overlapping, or adjacent marker intervals in a linkage group were treated as the same and given a common name as described by Ramya et al. (2010). The percentage of the phenotypic variation covered by QTL was estimated with the coefficient of determination $\left(\mathrm{R}^{2}\right)$ and for each QTL region were selected, was calculated the additive effect (Add). The effect of negative additive effect refers to cv. 'Magnat' while the positive effect to cv. 'Hewo'. 


\section{Results}

All traits had a normal distribution; the Shapiro-Wilk test as well as skewness and kurtosis analysis confirmed the proper distribution of all values for every experiment and each trait (Table 1). The ANOVA analysis showed that existed the significant differences among the genotypes $(p \leq 0.001)$ (Table 2$)$.

QTL analyses revealed 12 loci identified by a CIM, which appeared in at least two independent experiments and had LOD value $\geq 3.0$. These included: 3 stem length (STL), 2 plant high (PH), 1 spike length (SL), 2 number of spikelets per spike (NSS), 3 number of grains per spike (GS) and 1 thousand kernel weight (TKW) loci (Table 3).

\section{Stem length and plant height}

DH plants stem length (STL) observed in Experiments 1-4 ranged from $30.5 \mathrm{~cm}$ to 122.4 $\mathrm{cm}$; both extremes were noted in Experiment 4. The average STL of Experiment 1 carried out in the greenhouse was significantly lower than obtained in Experiments 2-4 conducted in the experimental plots (Table 1). The values of the plant height (PH) determined in Experiment 5 (STL and SL measured together) varied from 75 to $150 \mathrm{~cm}$. The Pearson's correlation showed high positive effect between within STL $(0.46,0.48$ and 0.53$)$ as well as between STL and SL $(0.52,0.55,0.65$ and 0.72 ; Table S1) values of each experiment. Furthermore, statistically significant high positive correlation was found between STL and/or PH and other measured traits within all experiments (Table S1).

Three STL QTLs and two PH QTLs were found on rye chromosome 5R (Table 3, Fig. S1, Fig. S2 - A). The most interesting locus, Qstl.hm.5R.1 detected in Experiments 1, 3, and 4, entirely overlaps Qph.hm.5R.1 from Experiment 5. Positive allele effect of both of those QTL regions referred to cv. 'Hewo' (Table 3). Regardless the experiments, the CIM analysis showed that those QTLs cover the same region of the 5R linkage group from 0 to $97.1 \mathrm{cM}$ position. That large QTL region might be resulted by the marker density of chromosome $5 \mathrm{R}$ as well as types of molecular markers used to develop the genetic map and length of that chromosome (247.1 cM; see Tyrka et at. 2015). It may indicate that 5R chromosome can be associated with controlling morphological traits. STL LOD valued reached over 20 (Table 3), and it explained from 56.5 to $60.4 \%$ of STL phenotypic variation, but $Q p h . h m .5 R .1$ was responsible only for $7.3 \%$ of $\mathrm{PH}$ variation. In each of the five experiments, the LOD peak was positioned at the same DArT marker $r P t$-390294-5R (Table 3).

The remaining STL QTLs were less expressed: Qstl.hm.5R.2 repeated in two experiments explained from 4.3 to $5.9 \%$ of the trait variation, respectively. It distinguished different, although nearly positioned markers in each of the experiments. The third STL QTL, Qstl.hm.5R.3, was found once in Experiment 4; however, interestingly, it entirely overlapped PH QTL Qph.hm.5R.2 from Experiment 5, having also the same LOD maximum and the same distinctive marker: $r P t-508323-5 R$ (Table 3). Negative allele effect of those QTL regions referred to cv. 'Magnat' (Table 3). 


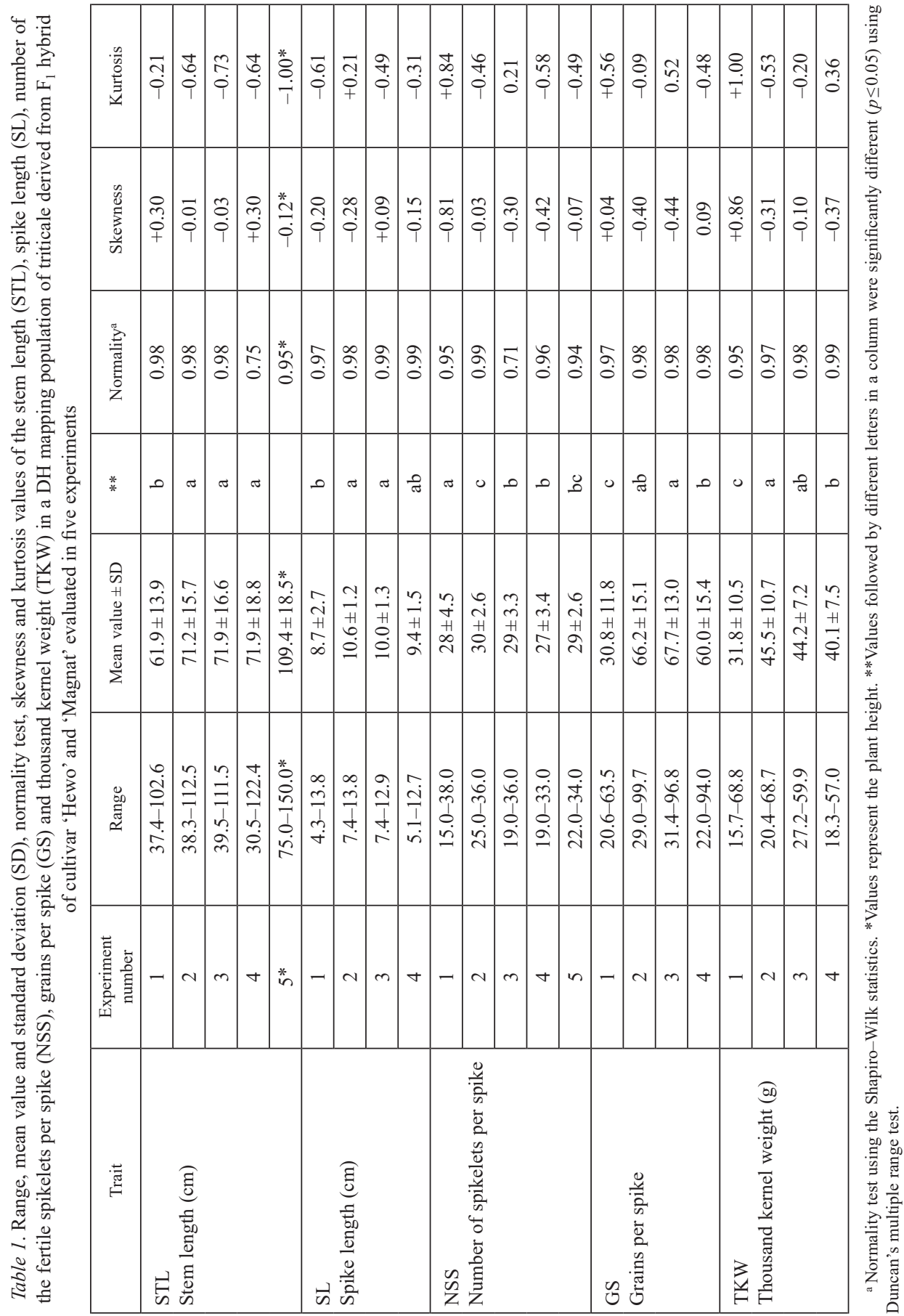




\section{Spike length}

SL ranged from $4.3 \mathrm{~cm}$ (Experiment 1) to $13.8 \mathrm{~cm}$ (Experiments 1 and 2) (Table 1). The shortest spikes were found under greenhouse conditions. Statistically significant high positive Pearson's correlation was found between SL within all four Experiments and NSS $(0.82,0.88,092$ and 0.95 at $0.001 \mathrm{P})$, GS $(0.72,0.82$ and 0.90 at $0.001 \mathrm{P})$ and TKW $(0.69,0.87$ and 0.93 at $0.01 \mathrm{P})$ values (Table $\mathrm{S} 1)$.

One SL QTL, Qsl.hm.6B.1, was identified on chromosome 6B and explained from 7.0 to 60.5 of the trait variability, depending on the experiment (Table 3, Fig. S1, Fig. S2 - B). That QTL in Experiments 1 and 2 was detected with LODs values 3.0 and 4.0 with the same distinctive marker: $3040572-6 B$ (Table 3). It is worth to noting that the spike length QTLs had an independent location from the stem length and the plant height QTLs. Negative allele effect of those QTL regions referred to cv. 'Magnat' (Table 3).

\section{Number of fertile spikelets per spike}

The NSS varied from 15 to 38, with both extremes found in Experiment 1. Averages are presented in Table 2. High positive Pearson's correlation, statistically significant was found between NSS within four out of five Experiments and GS (0.70, 0.78, 0.87 and $0.89)$ and TKW $(0.67,0.86$ and 0.88$)$ values (Table S1).

Two NSS QTLs were identified on chromosome 5R, with the LOD value varying between 5.6 and 11.3 (Table 3, Fig. S1, Fig. S2 - C). The average NNS was significant among 5 independent experiments (Table 1); however, QTLs were detected in Experiments 2, 4, and 5 exclusively. The NSS QTL Qnss.hm.5R.1 was identified twice (Experiments 2 and 4) and explained from 17.5 to $40.7 \%$ of the trait variation. It distinguished single e-SNP marker - 3612979. The second NSS QTL - Qnss.hm.5R.2, identified in Experiments 2 and 5, explained from 17.5 to $29.2 \%$ of the trait variation. It was only 3.5 $\mathrm{cM}$ in length, pointed to the same SNP marker (3604115) in both experiments and partly overlapped with Qstl.hm.5R.2 detected in Experiment 1, pointing to the same marker. Negative allele effect of those QTL regions referred to cv. 'Magnat' (Table 3).

\section{Grains per spike}

GS numbers ranged from 20.6 (Experiment 1) to 99.7 (Experiment 2). The average GS number differed significantly among experiments, with the distinctly lower number in the greenhouse conditions (Table 1). Statistically significant high positive Pearson's correlation was found between GS within all four Experiments and GS (0.70, 0.78, 0.87 and $0.89)$ and TKW $(0.67,0.86$ and 0.88$)$ values (Table S1).

The QTLs of GS were identified on wheat chromosomes 5A, 2B, and rye chromosome 5R (Table 3, Fig. S1, Fig. S2 - D, E, F). The Qgs.hm.5A.1, was located close to the DArT marker $w P t-1052-5 A$ in two out of five experiments and explained $11.66-16.80 \%$ of the phenotypic variation. Positive allele effect of this QTL region referred to cv. 'Hewo' (Table 3). The, Qgs.hm.5R.1, was identified close to DArT marker rPt-402162-5R 
(Experiment 2) as well as near e-SNP 4346981 and DArT 4346026-5R markers in the $4^{\text {th }}$ experiment and explained $7.0-44.83 \%$ of the phenotypic variation. Negative allele effect of this QTL referred to cv. 'Magnat' (Table 3).

Average spikelet fertility, i.e. the average number of grains produced by a single spikelet, was the lowest in the Experiment 1, and was equal to 1.1, while, in further experiments, it reached 2.2 in Experiments 2 and 4, and 2.3 in Experiment 3. QTLs for this trait were not detected.

\section{Thousand kernel weight}

The TKW value ranged from $15.7 \mathrm{~g}$ to $68.8 \mathrm{~g}$, and both extremes were noted in Experiment 1 (Table 1). The average TKW had the lowest value in the greenhouse conditions (Table 1). High positive Pearson's correlation, statistically significant was found between TKW within all four experiments and all measured traits (Table S1).

One locus associated with TKW was detected on rye chromosome 5R (Table 3, Fig. S1, Fig. S2 - G). Loci Qtkw.hm.5R.1 (maximum LOD 5.1 and 8.6 in Experiments 3 and 4,

Table 2. ANOVA of stem length (STL), spike length (SL), number of spikelets per spike (NSS), number of grains per spike (GS) and the thousand kernel weight (TKW) in a DH mapping population of triticale derived from $\mathrm{F}_{1}$ hybrid of cultivar 'Hewo' and 'Magnat' evaluated in five experiments. All values significant at $p \leq 0.001$

\begin{tabular}{|c|c|c|c|c|c|c|c|}
\hline Experiment & $\begin{array}{c}\text { Source } \\
\text { of variation }\end{array}$ & $\mathrm{df}$ & STL & SL & NSS & GS & TKW \\
\hline \multirow{3}{*}{1} & Genotypes & 88 & 777.042 & 16.783 & 80.277 & 553.568 & 447.199 \\
\hline & Block & 1 & 47.673 & 1.115 & 6.056 & 37.663 & 70.718 \\
\hline & Error & 88 & 255 & 255 & 252 & 252 & 252 \\
\hline \multirow{3}{*}{2} & Genotypes & 88 & 1068.583 & 6.188 & 33.241 & 941.003 & 477.164 \\
\hline & Block & 1 & 68.9256 & 0.478 & 4.143 & 79.674 & 34.109 \\
\hline & Error & 88 & 216 & 219 & 225 & 228 & 222 \\
\hline \multirow{3}{*}{3} & Genotypes & 88 & 1056.919 & 7.124 & 43.639 & 698.112 & 210.725 \\
\hline & Block & 1 & 25.779 & 0.696 & 2.975 & 87.008 & 17.626 \\
\hline & Error & 88 & 234 & 231 & 228 & 231 & 231 \\
\hline \multirow{3}{*}{4} & Genotypes & 88 & 1383.950 & 9.756 & 57.244 & 1097.161 & 3.585 \\
\hline & Blocks & 1 & 18.039 & 0.222 & 0.731 & 27.306 & 0.071 \\
\hline & Error & 88 & 258 & 216 & 246 & 249 & 249 \\
\hline \multirow{3}{*}{5} & Genotypes & 88 & 1096.854 & $\mathrm{n} / \mathrm{a}$ & 2.135 & $\mathrm{n} / \mathrm{a}$ & $\mathrm{n} / \mathrm{a}$ \\
\hline & Block & 1 & 103.056 & $\mathrm{n} / \mathrm{a}$ & 1.271 & $\mathrm{n} / \mathrm{a}$ & $\mathrm{n} / \mathrm{a}$ \\
\hline & Error & 88 & 225 & $\mathrm{n} / \mathrm{a}$ & 267 & $\mathrm{n} / \mathrm{a}$ & $\mathrm{n} / \mathrm{a}$ \\
\hline
\end{tabular}

$*_{\mathrm{n}} / \mathrm{a}-$ Not applicable. 


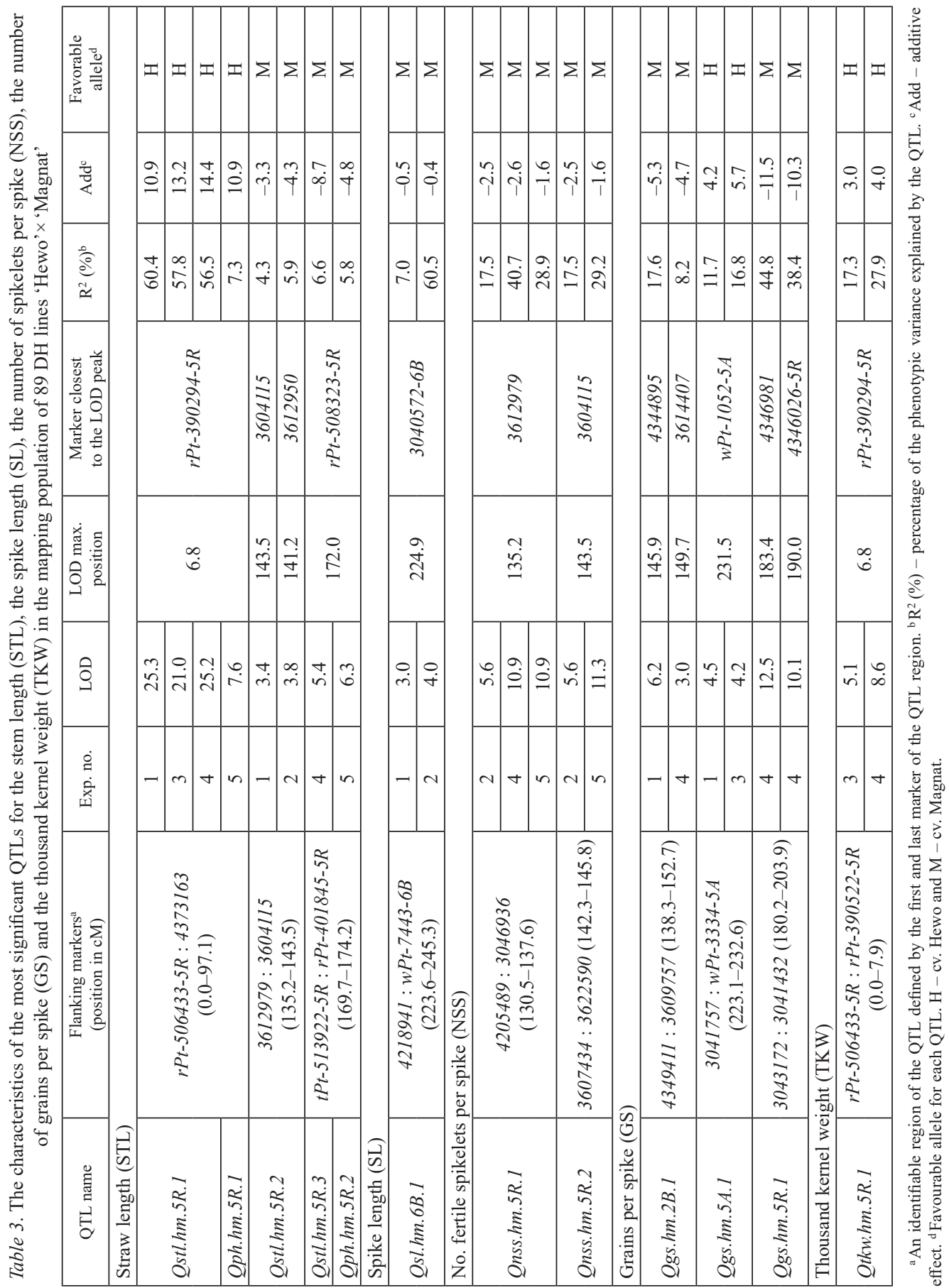


accordingly) had 0.0 to $7.9 \mathrm{cM}$ position, thus covered the first section of Qstl.hm.5R.1 and $Q p h . h m .5 R 1$, having also the same peak position and pointing to the same marker rPt-390294-5R. Positive allele effect of this locus refers to cv. 'Magnat' (Table 3).

\section{Discussion}

Phenotypic features recorded during present experiments showed large variation among lines and among experiments. Particularly, data from the Experiment 1, which was conducted in glasshouse conditions, revealed the presence of environmental stress, since the average STL, SL, GS, and TKW values were firmly below the data from Experiments $2-4$. They were also significantly lower in comparison to multi-year average values of these traits recorded in a gene bank between 1982 and 2008 years for Polish winter triticale (Banaszak 2011). Nevertheless, several QTLs identified in Experiment overlapped with QTL of the same features determined in other experiments, which point to strong genotypic control.

Genes actively controlling examined phenotypic features of DH population (STL, PH, SL, and NSS), were located mainly on chromosome 5R with the exception of SL, which was located on 5A and 6B chromosomes. The SL had no overlapping QTL, neither with STL nor with PH, although the latter included the sum of STL and SL values. This could indicate that the length of spike is regulated independently from the length of stem and could make possible independent breeding for the two features. The genetic control of morphological traits in small grain cereals has been studied by other authors, e.g., in maize (Peng et al. 2011), rice (Gao et al. 2016), barley (Mora et al. 2016), wheat (Ramya et al. 2010; Kumar et al. 2006, 2007; Li et al. 2002; Zhang et al. 2018) and rye (Miedaner et al. 2012).

Stem length in small grain cereals is an important and complex trait controlled by many genes (Miedaner et al. 2012; Würschum et al. 2014). In our study, three QTLs associated with STL were detected on rye chromosome 5R (Table 3, Fig. S1). The locus Qstl.hm.5R.1 detected nearby the marker $r P t-390294-5 R$ on the distal part of chromosome $5 \mathrm{R}$ in Experiments 1, 3, and 4, was a major QTL for this trait. It was stable and less affected by the environment, and explained 56.5 to $60.4 \%$ of phenotypic variation. This is in agreement with earlier results of Würschum et al. (2014), showing the QTL with the highest contribution to the genotypic variance on triticale chromosome 5R. Moreover, in our research, two QTLs associated with plant height (PH) in Experiment 5 were also detected on chromosome $5 \mathrm{R}$ and explained 5.8 to $7.3 \%$ of phenotypic variation. This result is comparable with the Alheit et al. (2014) report, which determined the major QTL for plant height on chromosome 5R as well. Furthermore, in rye, a major QTL of plant height was identified at the relative position on the chromosome comparable to the QTL identified in our research (Korzun et al. 1996; Börner et al. 1999). Additionally, dwarfing dominant gene $D d w 1$ located on rye chromosome 5R was successfully introduced into several triticale cultivars, including cv. 'Magnat' (Banaszak 2011), which considerably reduced plant height. Kalih et al. (2014) detected QTL associated with plant height at a similar position to Qph.hm.5R.1 identified in our experiments. Two remaining QTLs 
associated with STL (Qstl.hm.5R.2 and Qstl.hm.5R.3) on chromosome 5R individually explained less than $7 \%$ of the variation (Table 1). These results suggest that STL in triticale is a complex trait controlled by several major effect QTLs in addition to a few small effects QTLs. The molecular studies of other authors demonstrated that changes of genomic sequence, consisting of sequence elimination or the loss of the gene expression from the rye genome are common in triticale (Khalil et al. 2015).

In cereals, spike morphology is primarily determined by length, spikelet density, and the number of fertile floret, which are all controlled by polygenes. Spike length is an important component of spike morphology, which together with spikelet density can affect yield. In our study, one QTL region associated with SL was identified on wheat chromosome 6B (Table 3, Fig. S1). Locus Qsl.hm.6B.1, detected on chromosome 6B with closely located marker 3040572-6B, was significant for this trait with phenotypic variation up to 60.5 and LOD value 4.0 in Experiment 2 (Table 1). It indicates that this region on chromosome $6 \mathrm{~B}$ play an important role in determining spike length in triticale. In wheat, QTLs for this trait were also detected on chromosome 6B (Börner et al. 2002; Wang et al. 2011). However, Börner et al. (1999) documented QTL for SL on the long arm of chromosome $5 \mathrm{R}$ in rye.

Other traits important for yield are the number of spikelets per spike (NSS) and grain number per spike (GS), which is a complex trait, usually controlled by a number of QTLs with minor effects. There are no previous studies documenting QTL regions in triticale related to this trait. Only a few regions have been detected in wheat and rye (Kumar et al. 2007; Cui et al. 2012; Zhou et al. 2017). In our study, two QTLs associated with NSS were located on rye chromosome 5R (Table 3, Fig. S1). The QTLs were repeatedly identified in different experiments and found to be significant with a range of the phenotypic variation from $17.5 \%$ to $40.7 \%$. In rye, Börner et al. (1999) identified region on chromosome 5R locus, which could be related to morphological traits, including spike morphology, such as the length of the spike and number of spikelets per spike. In wheat, major NSS QTLs were identified on chromosomes 1A, 5A, and 7A (Kato et al. 2000; Zhou et al. 2017). On wheat chromosome 5A, Kato et al. (2000) identified QTL for yield traits, including spikelet number.

QTLs for GS have been previously reported on almost all wheat chromosomes, but the majority effects were only detected in single environments (Börner et al. 2002; Malyshev et al. 2003; Wang et al. 2011; Golba et al. 2013; Marcotuli et al. 2017; Zhou et al. 2017; Zhang et al. 2018). This trait also determines yield variability (Golba et al. 2013). In this study, three QTLs for GS were detected on chromosomes 5A, 2B, and 5R (Table 3, Fig. $\mathrm{S} 1$ ) with a range of phenotypic variation from 7.01 to $44.8 \%$. Identified Locus Qgs.hm.2B corresponded with the results of Marcotuli et al. (2017), where QTL for grain yield per spike was identified on chromosome $2 \mathrm{~B}$ candidate genes for this trait. Additionally, $\mathrm{Ku}-$ mar et al. (2007) detected two QTL regions associated with this trait on the short arm of chromosome 2B in bread wheat. Mangini et al. (2018) detect three QTLs for GS on chromosome 5A with significant effects on TKW suggesting that these QTL could represent genes involved in carbohydrate and/or storage protein synthesis with direct consequences on grain weight. 
Thousand kernel weight (TKW) is an important component of grain yield (Kumar et al. 2006; Mangini et al. 2018; Zhang et al. 2018). In wheat and rye, it is a trait controlled by a large number of QTL; however, little is known about the genetic control of this trait in triticale. In this study, one locus associated with TKW was detected on chromosome 5R (Table 3, Fig. S1) with the range of phenotypic variation from 17.3 to $27.9 \%$, depending on the experiment. Similarly, Liu et al. (2016) detected several QTLs associated with this trait, including loci on chromosome 5R with more than 5\% of the phenotypic variation. Similarly, a QTL for TKW in rye was detected on the long arm of chromosome 5R by Malyshev et al. (2003). In contrast, QTL associated with this trait in triticale have been previously reported by Wang et al. (2009) and Wang et al. (2011) on wheat chromosomes.

Interestingly, in this study, several QTLs for more than one trait were detected at the same position. Marker $r P t-390294-5 R$ was one of the associated markers flanking QTLs for STL and TKW traits with high LOD score (7.6-25.3 and 5.1-8.6, accordingly). The Pearson's correlation showed high positive effect for QTLs containing above marker (0.53, 0.56 and 0.81 ; Table S1). Additionally, the 3604115 marker was detected for STL and NSS traits with high LOD score (3.4 and 5.6-11.3, accordingly).

In conclusion, four different chromosomes from all of three triticale homologous genomes - wheat $(5 \mathrm{~A}, 2 \mathrm{~B}, 6 \mathrm{~B})$ and rye $(5 \mathrm{R})$, were involved in determining the selected yield components. Detected major QTLs associated with traits that determine triticale grain yield, might be useful for the identification of triticale genotypes in the process of breeding new varieties. The knowledge concerning the number and effects of QTLs would simplify marker-assisted selection and the development of cultivars with desirable characteristics.

\section{Acknowledgement}

The work was founded by the Polish National Science Centre (grant no. NN310 778 640).

\section{References}

Alheit, K.V., Busemayer, L., Liu, W, Maurer, H.P., Gowda, M., Hahn, V., Weissmann, S., Ruckelshausen, A., Reif, J.C., Würschum, T. 2014. Multiple-line cross QTL mapping for biomass yield and plant height in triticale (×Triticosecale Wittmack). Theor. Appl. Genet. 127:251-260.

Ammar, K., Mergoum, M,. Rajaram, S. 2004. The history and evolution of triticale. In: M. Mergoum (ed.) Triticale improvement and production. Fao Plant Production and Protection Paper 179.

Badea, A., Eudes, F., Salmon, D., Tuvesson, S., Vrolijk, A., Larsson, C.T., Caig, V., Huttner, E., Kilian, A., Laroche, A. 2011. Development and assessment of DArT markers in triticale. Theor. Appl. Genet. 122:1547-1560.

Banaszak, Z. 2011. Breeding of triticale in DANKO. Bericht über die 61:65-68.

Blum, A. 2014. The abiotic stress response and adaptation of triticale. Cereal Research Communications 42:359-375.

Börner, A., Korzun, A.V., Voylokov, A.V., Weberm, W.E. 1999. Detection of quantitative trait loci on chromosome 5R of rye (Secale cereale L.). Theor. Appl. Genet. 98:1087-1090.

Börner, A., Schumann, E., Furste, A., Coster, H., Leithold, B., Röder, M.S., Weber, W.E. 2002. Mapping of quantitative trait loci for agronomic important characters in hexaploid wheat (Triticum aestivum L.). Theor. Appl. Genet. 105:921-936. 
Cui, F., Ding, A., Li, J., Zhao, C., Wang, L., Wang, X., Qi, X., Li, X., Li, G., Gao, J., Wang, H. 2012. QTL detection of seven spike-related traits and their genetic correlations in wheat using two related RIL populations. Euphytica 186:177-192.

Gao, F., Zeng, L., Qiu, L., Lu, X., Ren, J., Wu, X., Su, X., Gao, Y., Ren, G. 2016. QTL mapping of grain appearance quality traits and grain weight using a recombinant inbred population in rice (Oryza sativa L.). J. Integr. Agric. 15:1693-1702.

Gawroński, P., Pawełkowicz, M., Tofil, K., Uszyński, G., Sharifova, S., Ahluvalia, S., Tyrka, M., Wędzony, M., Kilian, A., Bolibok-Brągoszewska, H. 2016. DArT markers effectively target gene space in the rye genome. Frontiers in Plant Science 7:1-13.

Golba, J., Rozbicki, J., Gozdowski, D., Sas, D., Madry, W., Piechocinski, M., Kurzyńska, L. Studnicki, M., Derejko, A. 2013. Adjusting yield components under different levels of $\mathrm{N}$ applications in winter wheat. International Journal of Plant Production 7(1):139-150.

Gołębiowska, G., Wędzony, M. 2009. Cold-hardening of winter triticale $(\times$ Triticosecale Wittm.) results in increased resistance to pink snow mould Microdochium nivale (Fr., Samuels \& Hallett) and genotypedependent chlorophyll fluorescence modulations. Acta Phys. Plant. 31:12-19.

Hoagland, D.R., Arnon, D.I. 1938. A water culture method for growing plants without soil. Circ. Univ. Calif. Agric. Exp. Stn. No. 347.

Kato, K., Miura, H., Sawada, S. 2000. Mapping QTLs controlling grain yield and its components on chromosome 5A of wheat. Theor. Appl. Genet. 101:1114-1121.

Kalih, R., Maurer, H.P., Hackauf, B., Miedaner, T. 2014. Effect of a rye dwarfing gene on plant height, heading stage and Fusarium head blight in triticale $(\times$ Triticosecale Wittmack). Theor. Appl. Genet. 127:1527-1536.

Khalil, H.B., Ehdaeivand, M.R., Xu, Y., Laroche, A., Gulick, P.J. 2015. Identification and characterization of rye genes not expressed in allohexaploid triticale. BMC genomics, 16:281.

Korzun, V., Börner, A., Melz, G. 1996. RFLP mapping of the dwarfing $(D d w 1)$ and hairy peduncle $(H p)$ genes on chromosome 5 of rye (Secale cereale L.). Theor. Appl. Genet. 92:1073-1077.

Kumar, N., Kulwal, P.L., Gaur, A., Tyagi, A.K., Khurana, J.P., Khurana, P., Balyan, H.S., Gupta, P.K. 2006. QTL analysis for grain weight in common wheat. Euphytica, 151(2):135-144.

Kumar, N., Kulwal, P.L., Balyan, H.S., Gupta, P.K. 2007. QTL mapping for yield and yield contributing traits in two mapping populations of bread wheat. Molecular Breeding 19(2):163-177.

Li, W.L., Nelson, J.C., Chu, C.Y., Shi, L.H., Huang, S.H., Liu, D.J. 2002. Chromosomal locations and genetic relationships of tiller and spike characters in wheat. Euphytica 125:357-366.

Liu, W., Leiser, W.L., Reif, J.C., Tucker, M.R., Losert, D., Weissmann, S., Hahn, V., Maurer, H.P., Würschum, T. 2016. Multiple-line cross QTL mapping for grain yield and thousand kernel weight in triticale. Plant Breeding 135:567-573.

Lukaszewski, A.J. 2003. Registration of three germplasms of hexaploid triticale with introgressions of wheat storage protein loci from chromosome 1D of bread wheat. Crop science 43:2316-2317.

Machczyńska, J., Orłowska, R., Mańkowski, D.R., Zimny, J., Bednarek, P.T. 2014. DNA methylation changes in triticale due to in vitro culture plant regeneration and consecutive reproduction. Plant Cell, Tissue and Organ Culture 119(2):289-299.

Malyshev, S.V., Kartel, N.A., Voylokov, A.V., Korzun, V., Börner, A. 2003. Comparative analysis of QTLs affecting agronomical traits in rye and wheat. In: Börner, A., Snape, J.W., Law, C.N. (eds) European Wheat Aneuploid Co-operative Newsletter. Proceedings of the $12^{\text {th }}$ EWAC Conference. Ss., pp. 120-122.

Mangini, G., Gadaleta, A., Colasuonno, P., Marcotuli, I., Signorile, A.M., Simeone, R., De Vita, P., Mastrangelo, A.M., Laidò, G., Pecchioni, N., Blanco, A. 2018. Genetic dissection of the relationships between grain yield components by genome-wide association mapping in a collection of tetraploid wheats. PLoS ONE 13(1): e0190162.

Marcotuli, I., Gadaleta, A., Mangini, G., Signorile, A.M., Zacheo, S.A., Blanco, A., Simeone, R., Colasuonno, P. 2017. Development of a high-density SNP-based linkage map and detection of QTL for $\beta$-glucans, protein content, grain yield per spike and heading time in durum wheat. International Journal of Molecular Sciences 18:1329. 
Miedaner, T., Hübner, M., Korzun, V., Schmiedchen, B., Bauer, E., Haseneyer, G., Wilde, P., Reif, J.C. 2012. Genetic architecture of complex agronomic traits examined in two testcross populations of rye (Secale cereale L.). BMC Genomics 13:706-719.

Mora, F., Quitral, Y.A., Matus, I., Russell, J., Waugh, R., del Pozo, A. 2016. SNP-Based QTL mapping of 15 complex traits in barley under rain-fed and well-watered conditions by a mixed modeling approach. Front. Plant Sci. 7:1-11.

Niedziela, A., Bednarek, P.T., Cichy, H., Budzianowski, G., Kilian, A., Anioł, A. 2012. Aluminum tolerance association mapping in triticale. BMC genomics 13:67.

Peng, B., Li, Y., Wang, Y., Liu, C., Liu, Z., Tan, W., Zhang, Y., Wang, D., Shi, Y., Sun, B., Song, Y., Wang, T., Li, Y. 2011. QTL analysis for yield components and kernel-related traits in maize across multi-environments. Theor. Appl. Genet. 122:1305-1320.

Ramya, P., Chaubal, A., Kulkarni, K., Gupta, L., Kadoo, N., Dhaliwal, H.S., Chhuneja, P., Lagu, M., Gupta, V. 2010. QTL mapping of 1000-kernel weight, kernel length, and kernel width in bread wheat (Triticum aestivum L.). J. Appl. Genet. 51:421-429.

Strang, E.J.P., Eklund, M., Rosenfelder, P., Sauer, N., Htoo, J.K., Mosenthin, R. 2016. Standardized ileal digestibility and basal ileal endogenous loss of amino acids associated with triticale genotypes in growing pigs. J. Animal Sci. 94:316-319.

Tyrka, M., Chełkowski, J. 2004. Enhancing the resistance of triticale by using genes from wheat and rye. J. Appl. Genet. 45:283-296.

Tyrka, M., Bednarek, P.T., Kilian, A., Wędzony, M., Hura, T., Bauer, E. 2011. Genetic map of triticale compiling DArT, SSR and AFLP markers. Genome 54:391-401.

Tyrka, M., Tyrka, D., Wędzony, M. 2015. Genetic map of triticale integrating microsatellite, DArT and SNP markers. PLoS ONE 10:1-17.

Tyrka, M., Oleszczuk, S., Rabiza-Swider, J., Wos, H., Wędzony, M., Zimny, J., Ponitka, A., ŚlusarkiewiczJarzina, A., Metzger, R.J., Baenziger, P.S., Lukaszewski, A.J. 2018. Populations of doubled haploids for genetic mapping in hexaploid winter triticale. Molecular Breeding 38(4):46.

Wang, R.X., Hai, L., Zhang, X.Y., You, G.X., Yan, C.S., Xiao, S.H. 2009. QTL mapping for grain filling rate and yield-related traits in RILs of the Chinese winter wheat population Heshangmai $\times$ Yu8679. Theor. Appl. Genet. 118:313-325.

Wang, S., Basten, C.J., Zeng, Z.B. 2007. Windows QTL Cartographer 2.5. Department of Statistics, North Carolina State University, Raleigh, NC. pp. 85.

Wang, J., Liu, W., Wang, H., Li, L., Wu, J., Yang, X., Li, X., Gao, A. 2011. QTL mapping of yield-related traits in the wheat germplasm 3228. Euphytica 177:277-292.

Würschum, T., Liu, W., Busemeyer, L., Tucker, M.R., Reif, J.C., Weissmann, E.A., Hahn, V., Ruckelshausen, A., Maurer, H.P. 2014. Mapping dynamic QTL for plant height in triticale. BMC Genetics 15:59.

Zhang, J., Gizaw, S.A., Bossolini, E., Hegarty, J., Howell, T., Carter, A.H., Akhunov, E., Dubcovsky, J. 2018. Identification and validation of QTL for grain yield and plant water status under contrasting water treatments in fall-sown spring wheats. Theoretical and Applied Genetics 131(8):1174-1759.

Zhou, Y., Conway, B., Miller, D., Marshall, D., Cooper, A., Murphy, P., Chao, S., Brown-Guedira, G., Costa, J. 2017. Quantitative trait loci mapping for spike characteristics in hexaploid wheat. The Plant Genome, 10:1-15.

\section{Electronic Supplementary Material (ESM)}

Electronic Supplementary Material (ESM) associated with this article can be found at the website of CRC at https://akademiai.com/loi/0806

Electronic Supplementary Table S1. Correlation coefficients between stem length (STL) and plant height (PH), spike length (SL), number of the fertile spikelets per spike (NSS), grains per spike (GS) and thousand kernel weight (TKW) in a DH mapping population of triticale derived from $\mathrm{F}_{1}$ hybrid of cultivar 'Hewo' and 'Magnat' evaluated in five experiments. Positive correlations are displayed in green and negative correlations in red color.

The color intensity is proportional to the correlation coefficients 
Electronic Supplementary Figure S1. Interval map (cM) of chromosomes 2B, 5A, 5R and 6B of 'Hewo' $\times$ 'Magnat' DH population of triticale ( $\times$ Triticosecale) with QTLs identified for morphological traits: stem length (STL), spike length (SL), number of spikelets per spike (NSS), grains per spike (GS) and thousand kernel weight (TKW). All QTLs were identified by SMA method. Additionally, black lines show markers identified by CIM method. The marks: $1^{\text {st }}, 2^{\text {nd }}, 3^{\text {rd }}, 4^{\text {th }}$ and $5^{\text {th }}$ correspond with the year of each experiment

Electronic Supplementary Figure S2. Results of the composite interval mapping (CIM) analysis performed for: A - stem length (STL), B - spike length (SL), C - number of spikelets per spike (NSS), D, F - grains per spike (GS) and $\mathrm{G}$ - thousand kernel weight (TKW). CIM profiles are presented on chromosomes 5A, 2B, 5B and 5R 